\title{
COLONIZACIÓN MICORRICICA EN PLANTAS DE AGUACATE (Persea americana L.)
}

\section{MYCORRHIZAL COLONIZATION IN AVOCADO PLANTS (Persea americana L.)}

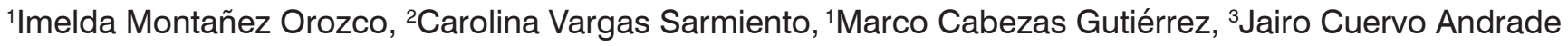

${ }^{1}$ Docentes Investigadores, Facultad de Ingeniería Agronómica. U.D.C.A. Dirección para correspondencia: Calle 222 No. 55-37, Bogotá, D.C. bmontanez@udca.edu.co; mcabezas@udca.edu.co. ${ }^{2}$ Microbióloga M.Sc. Docente Cátedra, Facultad de Ciencias Ambientales. U.D.C.A. Dirección para correspondencia: Calle 222 No. 55-37, Bogotá, D.C. martvargas@udca.edu.co. 3ingeniero Agrónomo Ph.D. Profesor Asociado, Facultad de Agronomía. Universidad Nacional de Colombia, Sede Bogotá. jlcuervoa@unal.edu.co.

Rev. U.D.CA Act. \& Div. Cient. 13 (2): 51-60, 2010

\section{RESUMEN}

Se realizó un estudio en plantas de aguacate en fase de vivero, para evaluar la colonización con hongos formadores de micorriza arbuscular (HFMA), utilizando tres suelos diferentes, procedentes de huertos, ubicados en el departamento del Meta, en los Llanos Orientales de Colombia. El objetivo fue determinar el tipo de colonización establecida empleando dos cepas nativas. Se aislaron, se identificaron y se propagaron dos morfotipos de HFMA pertenecientes a los géneros Glomus y Acaulospora, los cuales, se inocularon en tres variedades de aguacate: Lorena, Santana y Común. Se encontró que la colonización predominante para ambos fue de tipo Paris. El mayor porcentaje de colonización, se obtuvo en la variedad de aguacate Común, inoculada en un suelo tipo Hapludox, con el aislamiento de Glomus sp.

Palabras clave: Hongos formadores de micorriza, suelos ácidos, tipo de colonización, potencial de infectividad microbiana.

\section{SUMMARY}

In avocado plants, nursery phase, a study to evaluate the colonization with arbuscular mycorrhiza fungi (FMA) formers, using three different soils coming from orchard located at the eastern plains of Colombia was conducted. The objective was to determine the established colonization type using two native strains. Two morphotypes of FMA belonging to the genera Glomus and Acaulospora were isolated and identified and inoculated in three varieties of avocado Lorraine, Santana and Common. It was determined that the predominant colonization for both morphotypes was the Paris form. The highest colonization percentage was obtained in the Common avocado variety, inoculated in a Haplodux type soil with the Glomus isolation.

Key words: Fungi mycorrhiza formers, acidic soils, colonization type, potential microbial infectivity.

\section{INTRODUCCIÓN}

Conocido en el mundo como avocado, aguacate o aguacatero, la especie Persea americana L., actualmente, se produce en casi todos los países de climas cálido y templado, aunque los mayores cultivos están en países latinoamericanos, dentro de los que se destaca México, como primer productor mundial. En Colombia, este árbol puede crecer desde el nivel del mar hasta 2500m de altitud (Bernal E Díaz, 2005). De acuerdo con CORPOICA (2008), en algunos suelos de los Llanos Orientales, los rendimientos obtenidos son bajos (menores a $8 \mathrm{t} \cdot \mathrm{ha}^{-1}$ ), pero se pueden incrementar con un manejo tecnológico apropiado, el cual, parte de la nutrición mineral. Para lograr un adecuado balance de elementos nutricionales en el cultivo, se requiere la aplicación de enmiendas y fertilizantes, así como el uso de opciones orgánicas y biológicas, como el establecimiento de simbiosis micorrícicas, que contribuyan a reducir los impactos en el ambiente y a disminuir los costos de producción.

El término "micorriza " fue utilizado, por primera vez, por el Fitopatólogo alemán A. B. Frank, en 1885, para describir las estructuras modificadas de las raíces de especies forestales y, desde entonces, se ha extendido para referirse a un rango de asociaciones simbióticas mutualistas, entre hongos y raíces 
de plantas (Finlay, 2008). Se han distinguido siete categorías de simbiosis micorrícicas, con base en sus características morfológicas y las especies fúngicas y vegetales involucradas. La micorriza arbuscular es la forma más antigua y extendida, dado que la mayoría de las especies vegetales tienen la capacidad de formar esta asociación simbiótica (Redecker et al. 2000; Read, 2002).

Esta simbiosis, anteriormente recibió el nombre de "vesículo arbuscular", debido a las estructuras características formadas en la raíz de la planta hospedera. Las vesículas son estructuras fúngicas intracelulares de almacenamiento, que contienen lípidos y varios núcleos y actúan como propágalos. El hongo nunca entra en contacto con el citoplasma de las células de la planta hospedera; sin embargo, las imágenes de microscopia confocal, de raíces colonizadas muestran un aumento en el tamaño del núcleo de la célula vegetal, debido a la descondensación de la cromatina, pero no a la replicación del ADN y su centralización dentro de la misma (Barker et al. 1998; Sylvia et al. 1999).

Gracias a las observaciones e ilustraciones de las interacciones de los hongos con células y con tejidos de la raíz, realizadas por Gallaud en 1905 (Smith \& Read, 1997), se han clasificado las micorrizas arbusculares en dos grupos anatómicos, dependiendo de la especie vegetal asociada; estos dos patrones de colonización son el tipo Arum y el tipo Paris.

Muchas plantas herbáceas exhiben la colonización tipo Arum, la cual, involucra el crecimiento intercelular extensivo del hongo cuando éste penetra la corteza de la raíz, seguido después por la formación de arbúsculos. En esta asociación, el hongo se dispersa, de forma relativamente rápida, en la corteza de la raíz, mediante hifas, que se extienden a lo largo de los espacios de aire intercelulares. Las ramificaciones laterales cortas penetran las células corticales y se ramifican dicótomamente para formar los arbúsculos característicos. Se pueden formar enrollamientos o "coils" hifales, particularmente, en la capa de células hipodérmicas de la raíz, pero éstos no son un componente principal del micelio intraradical (Smith \& Read, 1997).

Un número similar de especies herbáceas presentan el patrón de colonización tipo Paris, en el cual, el crecimiento dentro de la raíz es lento, siendo, principalmente, intracelular, el hongo forma "coils" o enrollamientos, dentro de cada célula con muy pocos arbúsculos o mínimamente estructurados (Barker et al. 1998). Este tipo de colonización, se caracteriza por el desarrollo extensivo de hifas intracelulares enrolladas, que se extienden directamente de célula a célula dentro de la corteza. Los arbúsculos, se forman a partir de estos enrollamientos y existe muy poco o ningún crecimiento intercelular; como consecuencia, la velocidad de crecimiento de las unidades de infección dentro de las raíces es mucho más lenta que en la colonización tipo Arum (Smith \& Read, 1997). Gallaud describió este tipo de colonización en las especies forestales europeas de Parnassia sp. y Colchicum sp. y, también, ha sido descrito en miembros de la familia Gentianaceae y en varias especies forestales, como Erythronium sp., Trillium sp., Asarum sp., Acer saccharum, Liriodendron sp., Taxus sp., y Gingko sp. Además, se ha reportado en muchas familias de pteridófitas, gimnospermas y angiospermas (Smith \& Read, 1997).

En forma generalizada, se considera que los HFMA pueden mejorar el crecimiento de sus hospederos en suelos, donde el estado nutrimental es bajo. Así mismo, el efecto de la simbiosis y el establecimiento en el sistema radical son afectados por el estado nutrimental del suelo (Gianinazzi-Pearson \& Gianinazzi, 1981).

En vivero, se han tenido los mayores efectos en la implementación de la simbiosis de micorriza arbuscular, como una forma de mejorar la sanidad y el estado nutricional de las plantas, en la propagación de algunos frutales (Úsuga et al. 2008; Monticelli et al. 2000). En el caso de aguacate, ha sido reportado por Menge et al. (1980) y Godinez et al. (1986), un mayor crecimiento y sobrevivencia al trasplante en plantas micorrizadas versus no micorrizadas y, atribuyen a los HFMA, la mayor resistencia al estrés del trasplante, debido a que éstos mejoran la capacidad de absorción de agua (Pimienta-Barrios et al. 2009).

\section{MATERIALES Y MÉTODOS}

El ensayo, se realizó bajo cubierta en la Unidad DocenteInvestigativa El Remanso, de la Universidad de Ciencias Aplicadas y Ambientales U.D.C.A, que presenta una temperatura promedio de $22^{\circ} \mathrm{C}$., con variaciones diarias entre 14 y $30^{\circ} \mathrm{C}$. Los suelos utilizados en este estudio proceden de huertos de aguacate, ubicados en tres zonas diferentes del departamento del Meta: Centro Experimental Taluma, de la Corporación Colombiana de Investigación Agropecuaria (Corpoica), tipo Hapludox (S1); Puerto Colombia, tipo Udifluvents (S2) y Centro de Investigación La Libertad, de Corpoica, tipo Oxisol (S3), con base en la clasificación USDA (2010).

Con el fin de realizar el análisis fisicoquímico, se tomaron muestras de suelo, de acuerdo con la metodología propuesta por ICA (1992), enviadas al laboratorio de suelos del Instituto Geográfico Agustín Codazzi (IGAC). También, se colectaron muestras de suelo para el aislamiento, determinación del número y caracterización morfológica de esporas de HFMA. Este muestreo, se procedió tomando cuatro sub-muestras 
por árbol de aguacate (15 árboles por huerto), formando una cruz y siguiendo el borde del dosel. Los árboles tenían edad promedio de 15 años y estaban en estado de producción plena. Con un palín desinfectado, se hizo un corte de $15 \mathrm{~cm}$ de profundidad, $20 \mathrm{~cm}$ de ancho y $20 \mathrm{~cm}$ de largo; a este bloque, se le retiró el suelo asociado a raíces, se desmenuzó y se mezclaron las sub-muestras de suelo, para obtener una muestra compuesta de $500 \mathrm{~g}$, de cada uno de los árboles de aguacate.

Se utilizó un diseño de bloques completos al azar con arreglo factorial $3 \times 3 \times 3$, para un total de 27 tratamientos. La unidad experimental estaba representada por una planta obtenida a partir de semilla sexual. Se efectuaron ocho repeticiones, para conseguir, finalmente, 216 unidades experimentales. Los factores y los niveles de cada factor fueron los siguientes:

Factor A: $\quad$ Tres suelos diferentes: Taluma, Puerto Colombia y La Libertad.

Factor B: $\quad$ Tres variedades de aguacate: Lorena, Santana y Común.

Factor C. Inoculación con HFMA: Inoculado con Glomus sp., inoculado con Acaulospora sp. $\mathrm{y}$ tratamiento control o testigo (sin inocular).

Los suelos destinados, se sometieron a análisis físico y químico (IGAC, 1990) y, de acuerdo con los resultados (Tabla 1), se niveló el $\mathrm{pH}$ de todas las muestras a un mismo valor $(5,9)$ mediante encalado con cal dolomita. Con el propósito de homogenizar las condiciones nutricionales de los suelos, lo mismo que para garantizar el crecimiento de las plantas y favorecer el establecimiento de la simbiosis, se aplicó la mitad de la dosis de fósforo recomendada, equivalente a $12,5 \mathrm{~kg}$ ha-1 (Gómez, 2005), en forma de $\mathrm{P}_{2} \mathrm{O}_{5}$, para los suelos que presentaron bajo nivel de fósforo (Taluma y La Libertad).

Adicionalmente, a estos suelos se les practicó el proceso de solarización, con el fin de reducir la carga microbiana, incluyendo propágulos de HFMA. Este procedimiento, se llevó a cabo de acuerdo con la metodología probada por Gómez et al. (2000).

El material vegetal, se obtuvo mediante la selección de frutos de un mismo árbol procedente de un huerto, ubicado en el municipio de Fresno, departamento del Tolima, de ocho años de edad y en tercer año de producción plena. El huerto tiene 25 has, con la variedades Lorena, Santana y Común, en proporciones de $60 \%$, $25 \%$ y $15 \%$ de área plantada, respectivamente. Con el fin de reducir la heterogeneidad en el material propagado, las semillas fueron clasificadas por peso y se usaron las que estaban dentro del rango de 65 a $75 \mathrm{~g}$.

La selección de los géneros de HFMA, se estableció mediante la caracterización morfológica de esporas e identificación probable de las mismas, siguiendo las claves del INVAM (2009), a partir de las muestras de suelo, colectadas en los diferentes sitios de muestreo. En ellas, también se realizó el conteo de número de esporas por gramo de suelo y la cuantificación del porcentaje de colonización por HFMA en las raíces de árboles de aguacate, presentes en las muestras (Sieverding, 1983). Para la obtención de los inóculos, se efectuó la propagación empleando plantas de Brachiaria decumbens, inoculadas con esporas de los géneros de HFMA, predominantes en las muestras de suelo y comunes a las tres procedencias, siguiendo la metodología propuesta por Sieverding (1983).

La siembra, se desarrolló en la primera semana de agosto de 2008 , en bolsas plásticas con $5 \mathrm{~kg}$ de suelo; se colocó una semilla con el inóculo (seis esporas por planta), en el fondo del hoyo de siembra. El suelo, se mantuvo durante todo el ensayo a capacidad de campo. Las condiciones del invernadero, fueron: humedad relativa promedio de $74 \%$, medida con un hidro-termómetro marca GARMIN G2009 y radiación fotosintéticamente activa de $1320 \mu \mathrm{mol} \cdot \mathrm{m}^{-2} \cdot \mathrm{s}^{-1}$, registrada mediante un sensor cuántico marca LICOR L250A. A los siete meses después de la siembra, se determinó el porcentaje de

Tabla 1. Análisis de los tres suelos empleados en la investigación, colectados en diferentes localidades de los Llanos Orientales de Colombia.

\begin{tabular}{|c|c|c|c|}
\hline Suelo & $\begin{array}{c}\mathrm{pH} \\
\text { (en agua 1:1) }\end{array}$ & $\begin{array}{c}\text { Fósforo (ppm) } \\
\text { (Bray II) }\end{array}$ & Textura (Bouyoucos) \\
\hline Taluma & 4,4 & 7,3 & Arenoso Franco \\
\hline Puerto Colombia & 5,9 & 33,5 & Arenoso Franco \\
\hline La Libertad & 5,1 & No detectado & Arcilloso \\
\hline
\end{tabular}


colonización de raíces por HFMA y, para ello, se colectaron las muestras de raíces finas de 108 plantas de aguacate.

Para la determinación del porcentaje de colonización por HFMA en raíces de aguacate, se siguió la metodología de aclarado y de tinción de raíces, propuesto por Phillips $\varepsilon$ Hayman (1970), con algunas modificaciones: se reemplazó el colorante Azul de Trypan por tinta azul marca Parker ${ }^{\circledR}$, de acuerdo a la metodología propuesta por Vierheilig et al. (1998).

El análisis estadístico, se realizó mediante el programa SAS, versión 9.1 para Windows. Para dar mayor normalidad a los datos, se transformaron a $\sqrt{ } \mathrm{Y}+1$ y se efectuó el análisis de varianza, para cada variable. También, se practicaron pruebas de comparación múltiple, mediante el método Tukey.

\section{RESULTADOS Y DISCUSIÓN}

Los porcentajes de colonización por HFMA en raíces de aguacate presentaron diferencias estadísticamente significativas $(P<0,05)$, entre todos los tratamientos (Figura 1). Los mayores porcentajes de colonización correspondieron a los tratamientos inoculados; el más alto, se obtuvo con Acaulospora (82\%), seguido por Glomus (77\%) y, finalmente, el control (3\%). La colonización por HFMA en plantas testigo, se puede explicar por la persistencia de esporas en el suelo después del tratamiento de solarización. Smith \& Read (1997) afirman que en el suelo, las poblaciones de HFMA están compuestas de esporas de diferentes edades y en diferentes estados de dormancia o quiescencia.

Adicionalmente, es importante resaltar que la colonización de raíces por HFMA, se origina de tres fuentes de inóculo: esporas, fragmentos de raíz colonizados e hifas, las cuales, son denominadas, colectivamente, como propágulos (Gerdemann E Trappe, 1974; Gemma \& Koske, 1992; Lemus et al. 2005).

En cuanto a los suelos evaluados, según el análisis estadístico, existen diferencias significativas $(P<0,05)$ en el porcentaje de colonización de raíces, por HFMA (Figura 2). El mayor promedio, se obtuvo en el suelo de La Libertad (63\%), seguido por el suelo de Taluma (55\%) y, el menor, para el suelo de Puerto Colombia (45\%). El coeficiente de variación fue de $6,73 \%$, mientras que el $R^{2}$ fue 0,98 , lo que permite afirmar que estos resultados presentan alta confiabilidad y buen

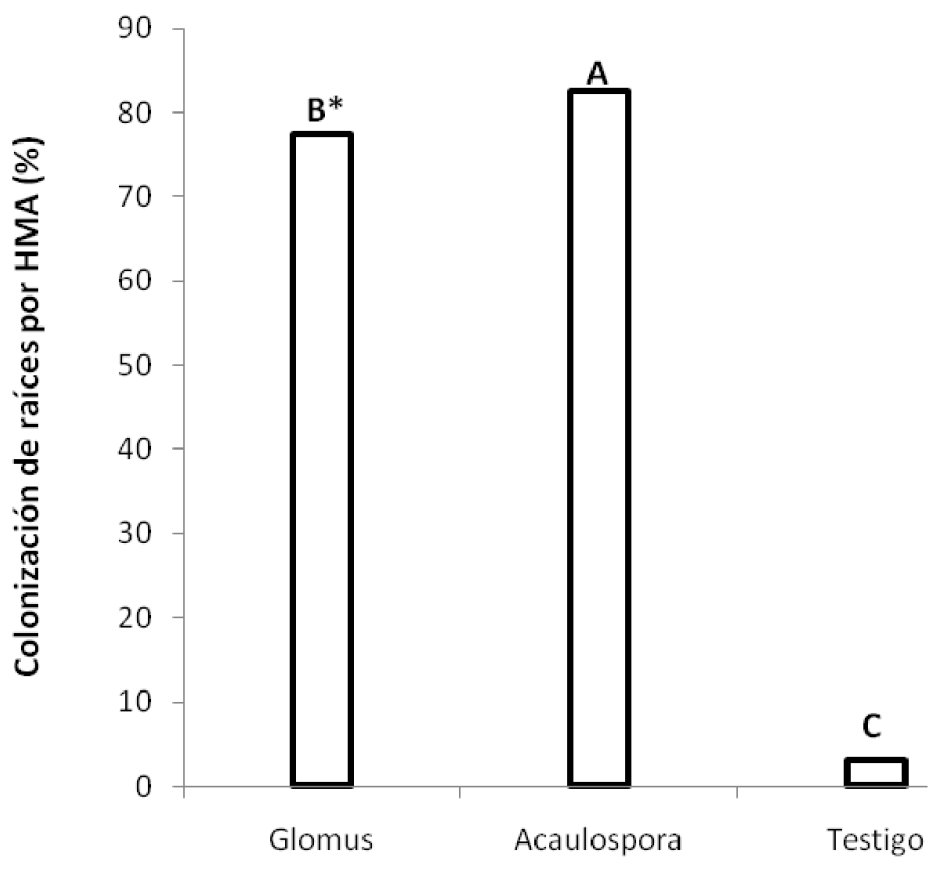

Tratamiento de inoculación

Figura 1. Porcentaje de colonización de raíces de aguacate (Persea americana L.), por hongos de micorriza arbuscular, por tratamiento de inoculación.

*Promedios seguidos de la misma letra no presentan diferencias estadísticamente significativas por la prueba de Tukey $(P \leq 0,05)$. 


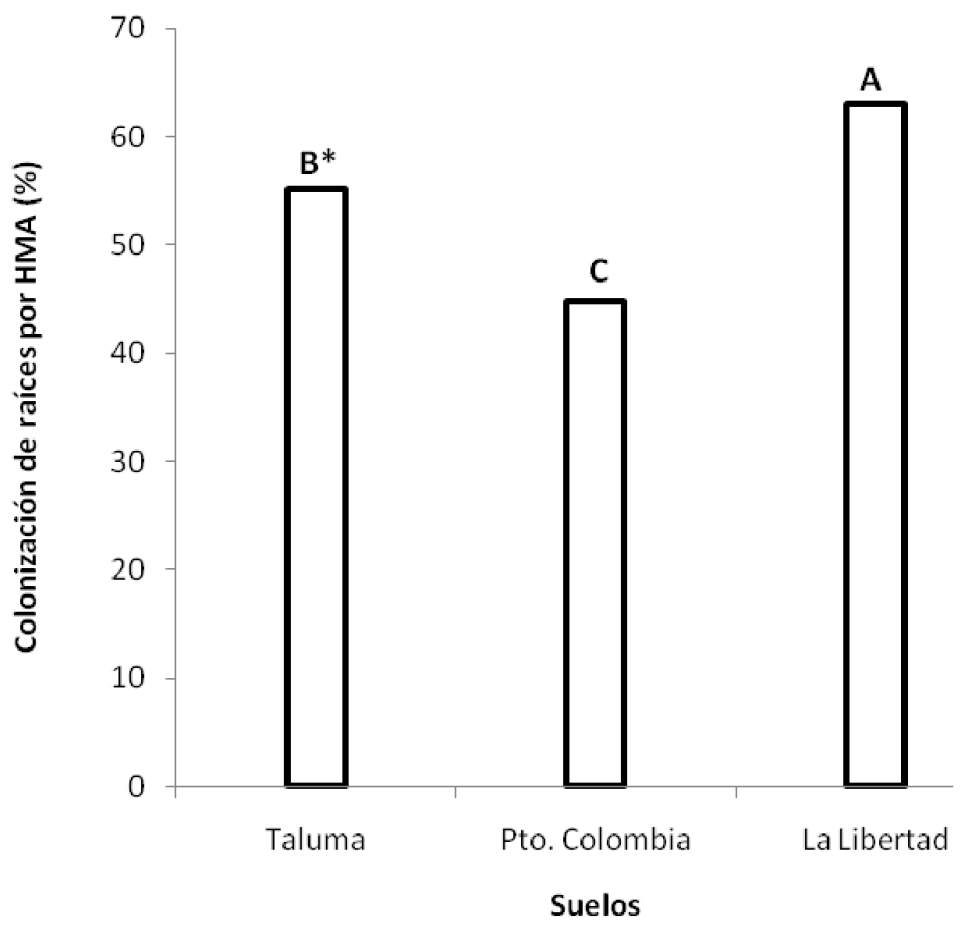

Figura 2. Porcentaje de colonización de raíces de aguacate (Persea americana L.), por hongos de micorriza arbuscular, para cada suelo evaluado. *Promedios seguidos de la misma letra no presentan evidencia de diferencias estadísticamente significativas, por la prueba de Tukey $(P \leq 0,05)$.

grado de homogeneidad. Este efecto puede estar relacionado con el contenido de fósforo en el suelo en condiciones naturales. Según los resultados del análisis de suelos, en Puerto Colombia, el nivel de fósforo es alto y el porcentaje de colonización determinado fue bajo, lo que concuerda con Rodríguez et al. (2002), quienes reportan que la micorrización es, generalmente, inhibida en suelos, con un alto contenido de fósforo.

En el suelo de La Libertad fue necesario aplicar una cantidad baja de fósforo, en forma de fertilizante, debido a la baja concentración natural del elemento. Esta condición pudo haber favorecido el establecimiento de la simbiosis, lo cual, se refleja en el alto porcentaje de colonización encontrado, aspecto que concuerda con lo expuesto por Roveda et al. (2007).

En cuanto a las variedades de aguacate, se observaron diferencias estadísticamente significativas $(P<0,05)$ en la colonización de raíces por HFMA (Figura 3). El mayor promedio se obtuvo en la variedad Común (58\%), seguido por la variedad Lorena (54\%) y, el menor, en la variedad Santana (51\%), lo cual, probablemente, se debe a que la colonización es regulada, tanto por la compatibilidad de los HFMA con las especies vegetales (Read, 2002) como por la presencia de poblaciones microbianas del suelo, que estimulan la germinación de esporas y la colonización de raíces de plantas hospederas, teniendo en cuenta que la solarización no elimina completamente las poblaciones de microorganismos del suelo y que, además, el crecimiento de las mismas está determinado por la presencia de las raíces. Así mismo, se debe considerar la existencia de microorganismos endófitos trasmitidos verticalmente, a través de las semillas, la que pueden influir sobre el establecimiento de otras relaciones simbióticas. Es posible, que la diferencia en los porcentajes de colonización entre los tratamientos, también esté determinada por las características de cada variedad de aguacate. Se conoce que la variedad Común presenta una alta adaptabilidad a diferentes condiciones ambientales, aspecto que correlaciona con la alta colonización (Amórtegui, 2001).

Al analizar estadísticamente las interacciones suelo-variedadinoculación, se percibió que el mayor promedio de porcentaje de colonización fue obtenido con la interacción suelo de Taluma-variedad Común-inoculada con Glomus con 98\%, que fue superior en $96 \%$, al tratamiento control; sin embargo, no se presentan diferencias significativas entre ésta y las 


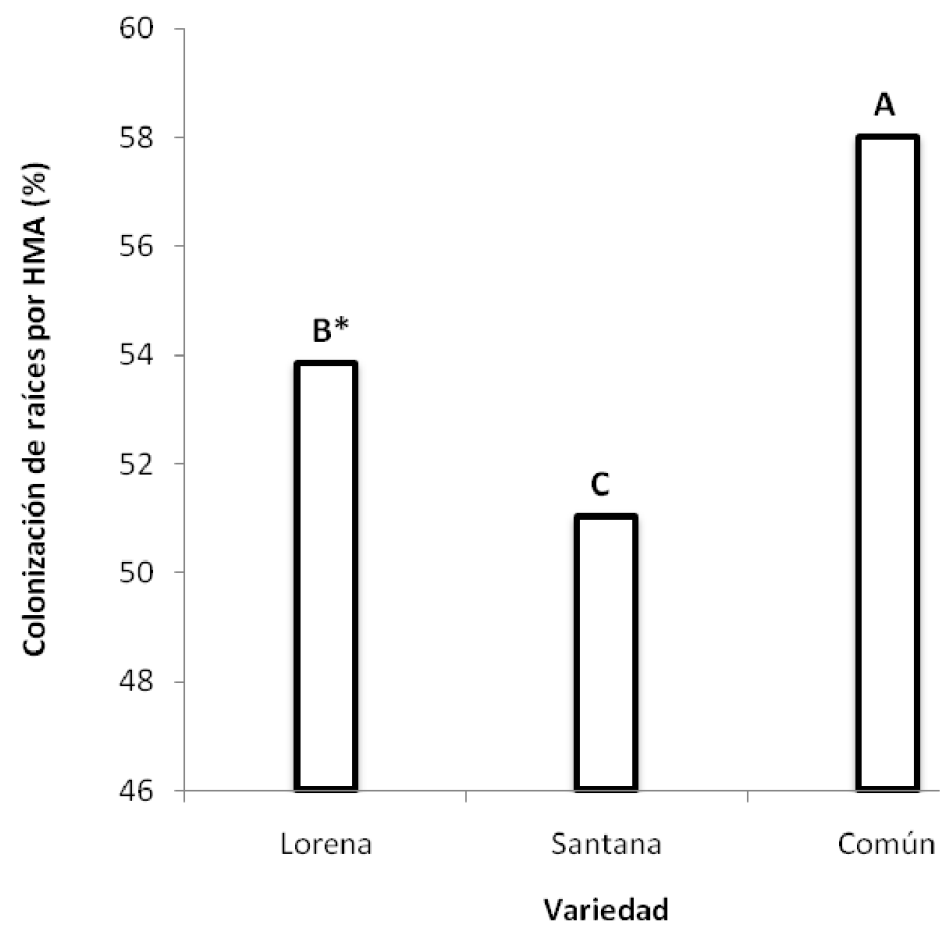

Figura 3. Porcentaje de colonización de raíces de diferentes materiales de aguacate (Persea americana L.), por hongos de micorriza arbuscular.

*Promedios seguidos de la misma letra no presentan diferencias estadísticamente significativas, por la prueba de Tukey $(P \leq 0,05)$.

interacciones La Libertad-Santana-Glomus y La LibertadComún-Acaulospora, con 97\%, las que son mayores al tratamiento control, en un $95 \%$.

Respecto a los promedios más bajos, se destaca que hay una correspondencia con los tratamientos sin inoculación con HFMA, siendo la interacción Taluma-Santana-Tratamiento control el tratamiento con promedio más bajo, $2 \%$, seguido de La Libertad-Común-Tratamiento control, 2\% y Taluma-ComúnTratamiento control, $2 \%$. Se resalta que los promedios más altos, se asocian con los tratamientos inoculados con HFMA.

En cuanto al tipo morfológico de colonización, se halló que las estructuras predominantes en todas las raíces de aguacate teñidas y observadas con el microscopio, corresponden con la colonización tipo Paris, la cual, se caracteriza por el desarrollo extensivo de hifas intracelulares, que se extienden, directamente, de célula a célula, dentro de la corteza de la raíz (Figura 4). En las muestras analizadas, no fue frecuente la presencia de estructuras típicas de la colonización micorrícica (arbúsculos y vesículas), por el contrario, los "coils" o enrollamientos intracelulares caracterizaron la colonización en la especie vegetal estudiada (Figura 4). Este hallazgo coincide con lo establecido por Armstrong E Peterson (2002), quienes señalan que en la colonización tipo Paris, los arbúsculos se extienden a partir del enrollamiento de hifas y el crecimiento intercelular es muy escaso o ausente.

La morfología de colonización, tipo Paris, consiste de "coils" o de enrollamientos intracelulares extensivos en las células corticales de la raíz; de estos enrollamientos, en ocasiones, se originan pequeños arbúsculos, pero no son lo suficientemente extensos para ser completamente responsables del intercambio de nutrientes. Se ha sugerido que la interface entre las células corticales y los enrollamientos intracelulares puede ser significativa en el intercambio de nutrientes (Harrison, 1999). En el presente estudio, se observaron estructuras consistentes con este tipo de colonización, cuya presencia en las raíces de las plantas estaría determinada por la interacción entre el genotipo de la planta y el del hongo micorrícico (Cavagnaro et al. 2001; Wu et al. 2004).

En las células corticales de las raíces de aguacate, también se encontraron estructuras correspondientes a hifas gruesas tabicadas de color marrón y esclerocios intracelulares que, de acuerdo con la literatura, pertenecen a hongos endófitos 

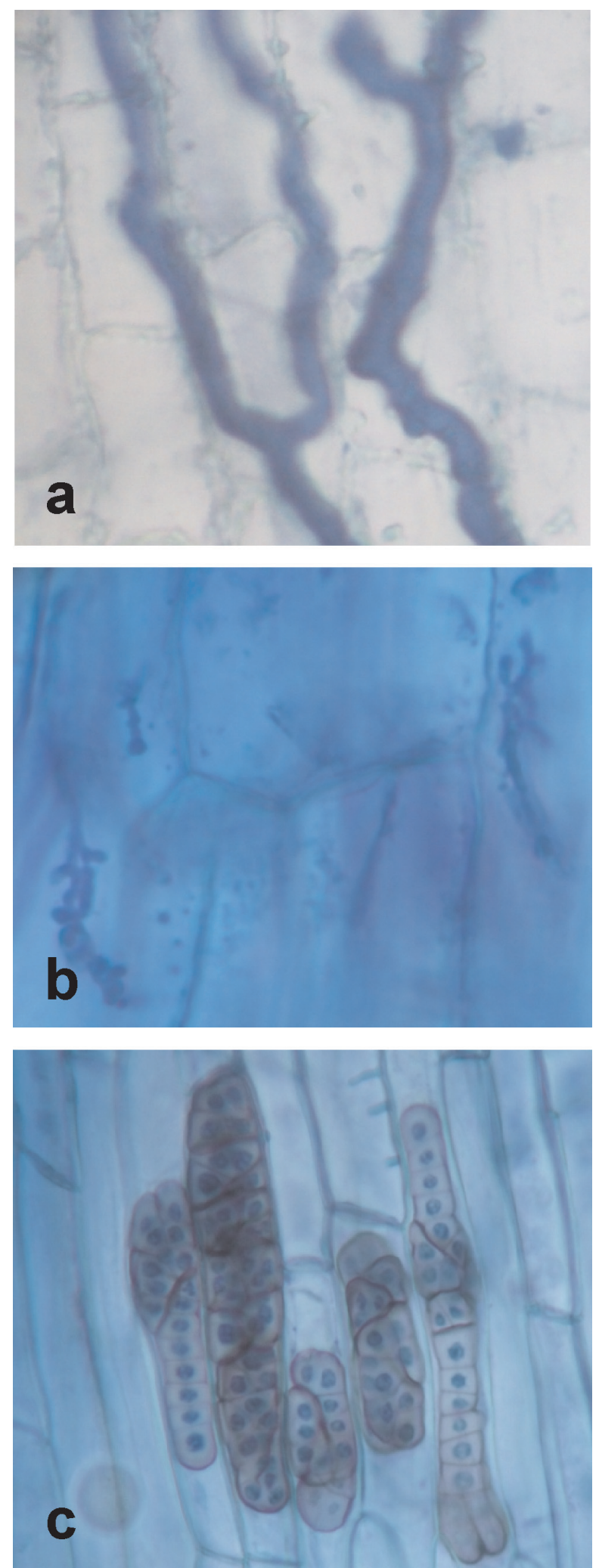

Figura 4. Aspectos sobresalientes de la colonización de raíces de plantas jóvenes de aguacate por HMA. a) Hifas de hongos formadores de micorriza arbuscular (HMA) en raíces de plantas jóvenes de aguacate; b) Coils o enrollamientos intracelulares característicos del tipo Paris de colonización por HMA y c) Esclerocios intracelulares de hongos endófitos septados y dematiáceos. 
septados dematiáceos u "oscuros", cuya presencia ha sido reportada en diversas especies vegetales (Urcelay, 2002; Urcelay et al. 2005). Los hongos endófitos septados oscuros dematiáceos son un grupo heterogéneo de Ascomycetes anamorfos, que pueden ser patógenos o benéficos para las plantas, dependiendo de la especie vegetal y el genotipo fúngico involucrados en la asociación (Jumpponen, 2001). Al presente, no existen estudios que hayan evaluado el efecto de estos simbiontes fúngicos sobre plantas de aguacate; sin embargo, la presencia de éstos en las plantas de aguacate estudiadas, no se relacionó con el desarrollo de enfermedad o algún síntoma visible de daño.

Bajo las condiciones de este estudio, se apreció una correlación negativa entre los contenidos de fósforo del suelo respecto al porcentaje de colonización de raíces por HFMA, de los géneros Glomus y Acaulospora. En cuanto al porcentaje de colonización de raíces por HFMA, la variedad Común se destaca por revelar los mayores promedios para diferentes interacciones de suelo y tratamiento de inoculación.

La colonización micorrícica de tipo Paris y la presencia de endófitos septados dematiáceos en raíces de aguacate reportadas en este estudio, sugiere que el espectro de asociaciones con simbiontes fúngicos en aguacate es amplio y genera nuevas expectativas, en cuanto al estudio de las relaciones filogenéticas y ecológicas, en las asociaciones micorrícicas, en esta especie.

Agradecimientos: Los autores agradecen a la Universidad de Ciencias Aplicadas y Ambientales U.D.C.A por la financiación del proyecto, así como también a la Facultad de Agronomía de la Universidad Nacional de Colombia, sede Bogotá. Conflicto de intereses: Este manuscrito fue preparado y revisado con la participación de todos los autores, quienes declaramos que no existe ningún conflicto de intereses que ponga en riesgo la validez de los resultados presentados.

\section{BIBLIOGRAFÍA}

1. AMÓRTEGUI, I. 2001. El cultivo del Aguacate. Módulo educativo para el desarrollo tecnológico de la comunidad rural. Corporación para el desarrollo rural y agroindustrial del Tolima - PROHACIENDO. 49p.

2. ARMSTRONG, L.; PETERSON, L. 2002. The interface between the arbuscular mycorrhizal fungus Glomus intraradices and root cells of Panax quinquefolius: a Parys - type mycorrhizal association. Mycologia. 94:587-595.
3. BARKER, S.; TAGU, D.; DELP, G. 1998. Regulation of root and fungal morphogenesis in mycorrhizal symbioses. Plant Physiol. 116:1201-1207.

4. BERNAL, J.; DÍAZ, C. 2005. Tecnología para el cultivo de aguacate. Manual Técnico 5. Corpoica. Centro de Investigación La Selva. Rionegro, Antioquia. 54p.

5. CAVAGNARO, T.; GAO, L.; SMITH, F.; SMITH, S. 2001. Morphology of arbuscular mycorrhizas is influenced by fungal identity. New Phytol. 151:469-475.

6. CORPOICA. 2008. Tecnología para el cultivo de aguacate. Bernal, J.; Tamayo, A.; Córdoba, O.; Londoño, M.; Tamayo, P.; Londoño, M. eds. Produmedios. Centro de Investigación La Selva Rionegro, Antioquia, Colombia. Manual Técnico 5. 241p.

7. FINLAY, R. 2008. Ecological aspects of mycorrhizal symbiosis: with special emphasis on the functional diversity of interactions involving the extraradical mycelium. J. Exp. Bot. 59(5):1115-1126.

8. GEMMA, J.; KOSKE, R. 1992. Are Mycorrhizal fungi present in early stages of primary succession? In: Read, D.J.; Lewis, D.H.; Fitter, A.H.; Alexander, I.J. eds. Mycorrhizas in Ecosystems. CAB International. Wallingford. U.K. p.183-189.

9. GERDEMANN, J.; TRAPPE, J. 1974. Endogonaceae in the Pacific Northwest. Mycologia Memoir. 5:1-76.

10. GIANINAZZI-PEARSON, V.; GIANINAZZI, S. 1981. Role of endomycorrhizal fungi in phosphorus cycling in the ecosystem. In: Wieklow, D.M.; Carroll, G.C. eds. The Fungal community, its organization and role in the ecosystem. p.637-652.

11. GODINEZ, R.; FERRERA-CERRATO, M.; CORTÉS, J.; DOMÍNGUEZ, J. 1986. Response of avocado (Persea americana Mill) to inoculation with endomycorrhiza V-A. Abstracts. Fourth International Simposium on Microbial Ecology. Ljubljana, Yugoslavia. August 24-29. p.150.

12. GÓMEZ, M. 2005. Guía técnica para el manejo nutricional de los cultivos. Microfertisa. Bogotá, D.C. 52p.

13. GÓMEZ, L.; GUERRA, D.; JARAMILLO, J.; BERNAL, J. 2000. Uso de la solarización como tratamiento de desinfección de suelo para semilleros de frutales y hortalizas en clima frío moderado. Memorias: Tercer seminario de frutales de clima frío moderado. Centro Tecnológico de Frutales C.D.T.F. Manizales, Colombia. p.105-111. 
14. HARRISON, M. 1999. Biotrophic interfaces and nutrient transport in plant - fungal symbioses. J. Exp. Bot. 50:1013-1022.

15. ICA. 1992. Fertilización en diversos cultivos. Quinta aproximación. Manual de Asistencia Técnica No. 25. Produmedios, Bogotá. 62p.

16. IGAC. 1990. Métodos Analíticos del Laboratorio de Suelos. 5a . Edición. Subdirección Agrológica. Bogotá, D.C. 189 p.

17. INVAM. 2009. Species Descriptions from Reference Cultures: Disponible desde internet en http://invam.caf. wvu.edu/fungi/taxonomy/speciesID.htm (con acceso 15/10/09).

18. JUMPPONEN, A. 2001. Dark septate endophytes - are they mycorrhizal. Mycorrhiza. 11:207-211.

19. LEMUS, G.; FERREYRA, E.; GIL, P.; MALDONADO, P.; TOLEDO, C.; BARRERA, C.; CELEDÓN DE ANDRACA, J. 2005. El cultivo del palto. Segunda edición. La Cruz, Chile. Instituto de Investigaciones Agropecuarias. Boletín INIA No 129. 76p.

20. MENGE, J.; LA RUE, J.; LABANAUSKAS, C.; JOHNSON, L. 1980. The effect of two mycorrhizal fungi upon growth and nutrition of avocado seedlings grown with six fertilizer treatments. J. Amer. Soc. Hort. Science. 105(3):400-404.

21. MONTICELLI, S.; PUPPI, G.; DAMIANO, C. 2000. Effects of in vivo mycorrhization on micropropagated fruit tree rootstocks. Applied Soil Ecology. 15(2):105-111.

22. PHILLIPS, J.; HAYMAN, D.1970. Improved procedures for clearing roots and staining parasitic and vesiculararbuscular mycorrhizal fungi for rapid assesment of infection. Transactions British Mycol. Soc. 55:158-161.

23. PIMIENTA-BARRIOS, E.; ZAÑUDO-HERNÁNDEZ, J.; LÓPEZ-ALCOCER, E. 2009. Efecto de las micorrizas arbusculares en el crecimiento, fotosíntesis y anatomía foliar de plantas jóvenes de Agave tequilana. Acta Botánica Mexicana 89:63-78.

24. READ, D. 2002. An ecological point of view on arbuscular mycorrhizal research. Gianinazzi, S.; Schüpp, H.; Barea, J.K.; Haselwandter, K. Mycorrhizal Technology in Agriculture. From genes to bioproducts. Berlin. p.129-136.
25. REDECKER, D.; KODNER, R.; GRAHAM, L. 2000. Glomalean fungi from the Ordovician. Science. 289:19201921.

26. RODRÍGUEZ, I.; CRESPO, G.; RODRÍGUEZ, C. 2002. Comportamiento de la macrofauna del suelo en pastizales con gramíneas naturales puras o intercaladas con Leucaena leucocephala para la ceba de toros. Rev. Cubana Ciencia Agrícola. 36(2):181-185.

27. ROVEDA, G.; CABRA, L.; RAMÍREZ, M.; PEÑARANDA, A. 2007. Efecto de las micorrizas arbusculares sobre la aclimatación y endurecimiento de microplántulas de mora (Rubus glaucus). Rev. Corpoica - Ciencia y Tecnol.Agropec. 8(1):28-36.

28. SIEVERDING, E. 1983. Manual de Métodos para la Investigación de la Micorriza Vesículo-arbuscular en el Laboratorio. CIAT. 271p.

29. SMITH, S.; READ, D. 1997. Mycorrhizal Symbiosis. Academic Press. Harcourt Brace $\&$ Company, Publishers, San Diego, London, New York, Boston, Sydney, Tokyo, Toronto. 605p.

30. SOIL SURVEY STAFF. 2010. Keys to Soil Taxonomy. United States Department of Agriculture Natural Resources Conservation Service. Eleventh Edition. 338p.

31. SYLVIA, D.; FUHRMANN, J.; HARTEL, P.; ZUBERER, D. 1999. Principles and Applications of Soil Microbiology. Prentice Hall, Upper Saddle River. New Jersey. E.U. 548p.

32. URCELAY, C. 2002. Co-ocurrence of three fungal root symbionts in Gaultheria oeppiggi DC in Central Argentina. Mycorrhiza. 12:89-92.

33. URCELAY, C.; TECCO, P.; CHIARINI, F. 2005. Micorrizas arbusculares del tipo 'Arum' y ‘Paris' y endófitos radicales septados oscuros en Miconia ioneura y Tibouchina paratropica (Melastomataceae). Bol. Soc. Argent. Bot. 40(3-4):251-255.

34. ÚSUGA, C.E.; CASTAÑEDA, D.A.; FRANCO, A.E. 2008. Multiplicación de hongos micorriza arbuscular (H.M.A) y efecto de la micorrización en plantas micropropagadas de banano (Musa AAA cv. Gran Enano) (Musaceae). Rev. Fac. Nal. Agr. Medellín. 61(1):4279-4290.

35. VIERHEILIG, H.; COUGHLAN, A.; WYSS, U.; PICHE, Y. 1998. Ink and vinegar, a simple staining technique for Arbuscular- Mycorrhizal fungi. Appl. Environm. Microbiol. 64(12):5004-5007. 
36. WU, B.; ISOBE, K.; ISHII, R. 2004. Arbuscular mycorrhizal colonization of the dominant plant species in primary successional volcanic deserts on the Southeast slope of Mount Fuji. Mycorrhiza. 14:391-395.

Recibido: Abril 30 de 2010

Aceptado: Noviembre 3 de 2010 